\title{
Distribution of bacterial communities along the spatial and environmental gradients from Bohai Sea to northern Yellow Sea
}

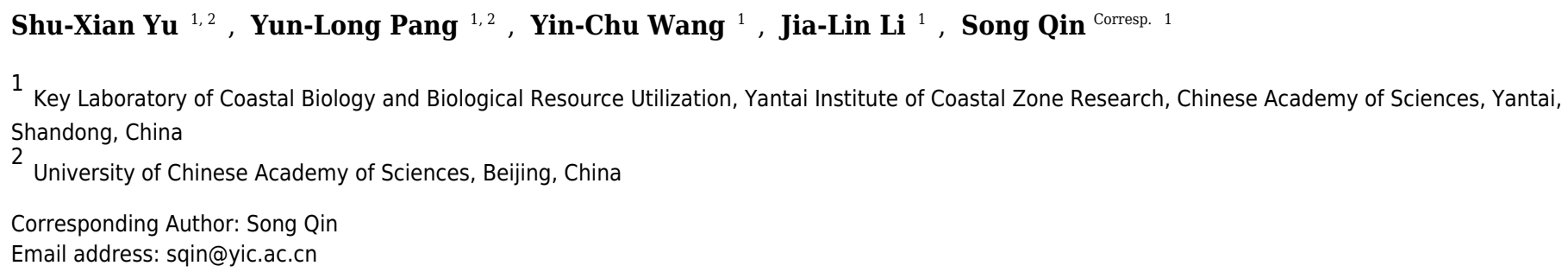

The eutrophic Bohai Sea receives large amount of suspended matters, nutrients and contaminant from terrestrial runoff, and exchanges waters with the northern Yellow Sea through a narrow strait. This coastal region provides an ideal model system to study microbial biogeography. We performed high-throughput sequencing to investigate spatial distribution of bacterial communities along the spatial and environmental gradients. The results showed bacterial communities presented remarkable horizontal and vertical distribution under coastal gradients. Fourteen abundant taxa clustered the samples into three distinctive groups, reflecting typical habitats in shallow coastal water (depth $\leq 20$ $m$ ), sunlit surface layer and bottom water (at 2-3 m above sediment). The most significant taxa of each cluster were determined by the least discriminant analysis effect size, and strongly correlated with spatial and environmental variables. Environmental factors (especially turbidity and nitrite) exhibited significant influences on surface bacterial betadiversity, while community similarity in bottom water was mainly determined by depth. Geographic distance enhanced bacterial community dissimilarity and limited bacteria distribution in both surface and bottom water. Bacterial dispersal was also constrained by phosphate, which was possible due to the high ratios of N/P in this coastal sea area. 
1 Distribution of bacterial communities along the spatial and environmental gradients from Bohai

2 Sea to northern Yellow Sea

3 Shu-Xian Yu ${ }^{1,2}$, Yun-Long Pang ${ }^{1,2}$, Yin-Chu Wang ${ }^{1}$, Jia-Lin Li ${ }^{1}$, Song Qin ${ }^{1, *}$

$4{ }^{1}$ Key Laboratory of Coastal Biology and Biological Resource Utilization, Yantai Institute of

5 Coastal Zone Research, Chinese Academy of Sciences, Yantai 264003, China

$6{ }^{2}$ University of Chinese Academy of Sciences, Beijing 100049, China

7 *Corresponding author: Song Qin, email: sqin@yic.ac.cn 


\section{Abstract}

9 The eutrophic Bohai Sea receives large amount of suspended material, nutrients and contaminant

10 from terrestrial runoff, and exchanges waters with the northern Yellow Sea through a narrow

11 strait. This coastal region provides an ideal model system to study microbial biogeography. We

12 performed high-throughput sequencing to investigate the distribution of bacterial taxa along

13 spatial and environmental gradients. The results showed bacterial communities presented

14 remarkable horizontal and vertical distribution under coastal gradients of spatial and

15 environmental factors. Fourteen abundant taxa clustered the samples into three distinctive groups,

16 reflecting typical habitats in shallow coastal water (seafloor depth $\leq 20 \mathrm{~m}$ ), sunlit surface layer (at

17 water surface with seafloor depth $>20 \mathrm{~m}$ ) and bottom water (at 2-3 m above sediment with

18 seafloor depth $>20 \mathrm{~m}$ ). The most significant taxa of each cluster were determined by the least

19 discriminant analysis effect size, and strongly correlated with spatial and environmental

20 variables. Environmental factors (especially turbidity and nitrite) exhibited significant influences

21 on bacterial beta-diversity in surface water (at $0 \mathrm{~m}$ sampling depth), while community similarity

22 in bottom water (at 2-3 m above sediment) was mainly determined by depth. In both surface and

23 bottom water, we found bacterial community similarity and the number of OTUs shared between

24 every two sites decreased with increasing geographic distance. Bacterial dispersal was also

25 affected by phosphate, which was possible due to the high ratios of IN/IP in this coastal sea area. 
26

27

28

29

30

31

\section{Introduction}

Microbes are recognized as the vital biological engines that drive global biogeochemical cycling (Falkowski et al. 2008). To better understand the microbial roles in biogeochemical processes, an indispensable subject is to unravel the mechanisms of microbial organization and succession over space and time in natural ecosystems (van der Gast 2013). According to Martiny et al. (2006), microbial biogeography can be determined by the influences derived from both contemporary environmental conditions and historical contingencies, which correspond to the active and passive reactions of microorganisms facing ever-shifting environment and geographic separation.

The relative influence of environmental factors and dispersal limitation brought to bear onto microbial diversity is likely dependent on spatial scale (Martiny et al. 2011). At small spatial scales within a few kilometers, environmental conditions are frequently detected as the determinant forces affecting microbial composition, while the distance effects seem to be negligible; geographic separation can overwhelm any environmental effect at global scale; within an intermediate scale of ten to thousands of kilometers, both environmental factors and geographic distance are likely detected to affect community composition (Martiny et al. 2006).

However, actual situations could be very complicated in natural ecosystems, because effects of local environment and historical factors are not always possible to be separated clearly (Lindstrom \& Langenheder 2012). Particularly in coastal waters, natural ecosystems are subject to intense interactions between terrestrial and oceanic processes, as well as frequent disturbance from human activities (such as pollution and eutrophication), resulting in regional heterogeneity of physicochemical conditions (Crossland et al. 2005). When physicochemical factors are 
47 spatially autocorrelated, spatial effects may superficially mask environmental effects. However,

48 persistent environmental heterogeneity is in fact possible to overwhelm geographic separation in

49 generating and maintaining microbial diversity at intermediate spatial scales (Wang et al. 2015).

50 In addition, water depth is another noteworthy factor that can shape vertical distribution of

51 microbes in coastal seas (Zinger et al. 2011). Gong et al. (2015) have identified that water depth

52 was the decisive factor to shape biogeography pattern of benthic microbial eukaryotes in the

53 surface sediments of coastal sea. Our previous study (Li et al. 2014) also displayed that water

54 depth played a surprisingly significant role in shaping bacterial biogeography in the upper 5

meters of water column characterized with complex circulation systems. We consider that water

56 depth may function as a comprehensive proxy of multiple physicochemical parameters rather

57 than directly affect microbial diversity. Furthermore, coastal sea is usually defined as the seaward

area with water depth $<200$ m (Pernetta \& Milliman 1995); such shallow depth may allow

microbes frequently exchange through water column, and then contribute to microbial ecological

60 shift in both surface and bottom layers of coastal waters. However, this issue could not be

61 confirmed due to the lack of samples derived from bottom layer in our previous study.

62 Therefore the driving forces, including physicochemical factors, geographic distance and water

depth, should be thoroughly considered when investigating microbial biogeography in coastal

waters. Owing to pervasive applications of molecular approaches, especially high-throughput 
69

contributions of major driving forces to microbial diversity in under-surface layers of shallow

coastal water (seafloor depth $<200 \mathrm{~m}$ ).

Here we choose the sea area from Bohai Sea to northern Yellow Sea (Fig. 1) to investigate the spatial distribution of bacterioplankton. The Bohai Sea is an almost-enclosed sea area, where the average depth is $18 \mathrm{~m}$ and the water residence time is about 11-12 years (Lee et al. 2002). In contrast, the Yellow Sea is relatively a more open region located on the continental shelf, with an average depth of $44 \mathrm{~m}$ and a residence time of 5-6 years (Lee et al. 2002; Zheng et al. 2006). The Bohai Sea and Yellow Sea can exchange sea water through a sole narrow strait. The study area has distinct seasonal characteristics; in summer, most of this area is clearly stratified, while the northwesterly monsoon destroys density stratification in winter (Lin et al. 2006; Su 2001).

Additionally, the Yellow River discharges a large amount of sediments and contaminants into the southern part of Bohai Sea. In 2008, about $19 \times 10^{3}$ tons of ammonium from Yellow River were loaded into the Bohai Sea, causing an average N/P ratio of 67 in the entire Bohai Sea (Dang et al. 2013). Increasing $\mathrm{N} / \mathrm{P}$ ratio has been considered as an important factor to determine the dominant phytoplankton composition in the Bohai Sea shifting from diatoms to dinoflagellates during the last decades (Wei et al. 2004). In general, physicochemical conditions, e.g. temperature, salinity and nutrients, exhibit gradient distribution patterns in the surface and bottom water from Bohai Sea to northern Yellow Sea (Chen 2009). Accordingly, this coastal region is an ideal model system to study the microbial distribution along spatial and physicochemical gradients, as well as to unveil relative influences of water depth, geographic distance and environmental factors. Therefore, our specific goals were to determine: (i) whether the dominant composition of bacterial communities varies along spatial and physicochemical gradients in this region; and (ii) 
91 whether geographic distance and depth contribute more than environmental factors to the spatial

92 variation of microbial communities in both surface and bottom water.

93 Materials and Methods

94 Sampling and environmental parameters

95 The sampling sites were determined along the center axis from the edge of Yellow River Estuary

96 to northern Yellow Sea. Water collection was performed at seven stations (Fig. 1) during the open

97 research cruise of the Bohai Sea and northern Yellow Sea in August/September of 2014. At each

98 site, two liters of seawater was collected from the surface layer $(0 \mathrm{~m})$ and the bottom layer $(2-3$

$99 \mathrm{~m}$ above sediment), and then filtered through $0.22 \mu \mathrm{m}$ pore-size white polycarbonate filters.

100 These filters were immediately sealed in sterile $50 \mathrm{~mL}$ centrifuge tubes and stored at $-80^{\circ} \mathrm{C}$ for

101 further DNA extraction.

102 Environmental parameters, including temperature, salinity and depth, were measured in situ using

103 a CTD sensor (Sea-Bird Electronics Inc., USA). We measured pH, turbidity, dissolved oxygen

104 (DO) and chlorophyll $a(\mathrm{Chl} a)$ on boat with a probe (Hydrolab MS5, HACH, USA).

105 Concentrations of ammonium, nitrite, nitrate, phosphate and silicate were measured by a nutrient

106 flow analyzer (Seal, Germany).

107 DNA extraction, PCR amplification and high-throughput sequencing

108 The total DNA on filters was extracted using the FastDNA SPIN Kit for soil (MP BIO, USA)

109 according to the manufacturer's instruction. The quality of the DNA extracts was determined by

110 agarose gel electrophoresis. We used a NanoDrop 2000c spectrophotometer (ThermoFisher,

111 USA) to measure the DNA concentration. 
112 The universe prokaryotic primers 515F (5'-GTG CCA GCM GCC GCG GTA A-3') and 907R (5'-

113 CCG TCA ATT CCT TTG AGT TT-3') were used to target the V4-V5 region of 16S rRNA genes.

114 Triplicate PCRs were carried out by ABI GeneAmp 9700, using purified DNA as a template. The

115 PCR process started with a pre-denaturation period at $95^{\circ} \mathrm{C}$ for $5 \mathrm{~min}$, followed by 35 cycles of

116 denaturation at $94{ }^{\circ} \mathrm{C}$ for $30 \mathrm{~s}$, annealing at $55^{\circ} \mathrm{C}$ for $30 \mathrm{~s}$ and elongation at $72{ }^{\circ} \mathrm{C}$ for $40 \mathrm{~s}$, and

117 ended with a final extension at $72{ }^{\circ} \mathrm{C}$ for $7 \mathrm{~min}$.

118 PCR products were purified by agarose gel electrophoresis with an AxyPrep DNA Gel Extraction

Kit (Axygen, USA), and then quantified with a QuantiFluor-ST Fluorometers (Promega, USA).

High-throughput sequencing was performed at Majorbio Co., Ltd. (Shanghai, China) using the

250-bp PE Illumina MiSeq sequencing platform.

\section{Data processing and statistical analysis}

Raw sequence profiles were processed using QIIME (Caporaso et al. 2010b), and analyzed as

previously described by Wang et al. (2015) with several modifications. In brief, retained highquality reads need to meet the criterions of Phred quality score > 20 (Q20), no ambiguous bases

(no N's), and consecutive high-quality bases $>80 \%$ of total read length. Chimeras were filtered

the remaining sequences based on a 97\% sequence similarity with the UCLUST method (Edgar, 
134 less than $0.01 \%$ of total sequences (Bokulich et al. 2013). Eventually, a total of 598261 clean

135 reads and 481 bacterial OTUs were obtained from 14 samples.

136 To estimate biodiversity of bacterial communities, we used the core_diversity_analyses.py script

137 to calculate alpha-diversity indexes and beta-diversity distance with an evenly sequencing depth

138 of 10,000 reads per sample. Multiple indices for alpha-diversity of bacterial communities were

139 generated, including observed OTUs, Good's coverage, phylogenetic diversity, Chao1 and

140 Shannon-Wiener index. Weighted UniFrac distance was calculated to reveal beta-diversity.

141 Principal coordinate analysis (PCoA) was performed to visualize community dissimilarities

142 (beta-diversity) using R v.3.3.1 (R Development Core Team 2008).

143 The dominant phyla, which had a relative abundance $>1 \%$ in at least one sample, were utilized

144 when making heat maps and conducting hierarchical clustering analysis (HCA). The discriminant

145 taxa from each major cluster (suggested by HCA) were determined by the LDA (least

146 discriminant analysis) effective size (LEfSe), using Kruskal-Wallis sum-rank test $(\alpha=0.05)$ and

147 one-against-all comparisons (Segata et al. 2011). Pearson's and Spearman's correlations between

148 variables were calculated by R program. One-way analysis of variation (ANOVA) and least

149 significance difference test (LSD, 0.5 confidence threshold) were conducted to determine the

150 environment and community dissimilarity between surface and bottom layers. Pairwise

151 geographic distances were generated from the longitude and latitude of each site with R program.

152 Mantel and partial Mantel tests (with 999 permutations) (Legendre \& Legendre 2012) were

153 conducted to unveil the relationships between environment (physicochemical factors), geographic

154 distance, depth and beta-diversity (weighted UniFrac distance). For all correlation tests in this

155 study, Benjamini-Hochberg multiple test correction was performed to control the false discovery 
156 rate of $\mathrm{P}$ values. To reveal spatial separation, we simulated general linear model (GLM) in scatter

157 plots of geographic distance, water depth, environmental variables and community similarity

158 (calculated as 1-weighted UniFrac distance or numbers of pairwise shared OTUs).

159 Results

160 Spatial variation of physicochemical parameters

161 We measured environmental parameters and calculated Pearson's correlations between

162 physicochemical parameters and spatial factors (longitude, latitude, water depth and collection

163 depth; Dataset S1). In surface water of P1, the measured turbidity and salinity were 3.19 ntu and

$16430.05 \mathrm{~g} \mathrm{~L}^{-1}$ (Dataset S1a), indicating that P1 was near to the Yellow River Estuary in comparison

165 with previous records (Shen et al. 2010; Zhang et al. 2014). Longitude showed a positive

166 relationship with water depth $(\rho=0.91, \mathrm{P}<0.01$; Dataset $\mathrm{S} 1 \mathrm{~b})$, which is consistent with the

167 topographic feature that water depth generally increases from the shallow coastal site P1 to the

168 northern Yellow Sea site K5. With increasing longitude and water depth, the sampling site was

169 further from the shallow coast and estuary. Thus, the negative relationship between longitude,

170 water depth and turbidity $(\rho=-0.71, \mathrm{P}<0.01 ; \rho=-0.6, \mathrm{P}=0.02$; Dataset S1b; Fig. S1) was

171 reasonable to understand. In addition, salinity also presented remarkable spatial autocorrelation

172 (longitude, $\rho=0.83, \mathrm{P}<0.01$; Dataset S1b; Fig. S1). Distributions of ammonium, nitrite, nitrate

173 and phosphate were not detected to be prominently related to longitude and latitude (Dataset S1b-

174 d), whereas the ratios of inorganic Nitrogen to Phosphorus (IN/IP) ranged from 10 to 152, with

175 an average value of 55 (Dataset S1a). Collection depth was significantly correlated with

176 temperature $(\rho=-0.91, \mathrm{P}<0.01)$, salinity $(\rho=0.68, \mathrm{P}=0.01), \mathrm{pH}(\rho=-0.76, \mathrm{P}<0.01)$, nitrate 
$177(\rho=0.6, P=0.02)$, phosphate $(\rho=0.81, P<0.01)$ and silicate $(\rho=0.77, P<0.01)$, suggesting

178 distinct environmental conditions between surface and bottom water. One-way ANOVA and LSD

179 test confirmed the dissimilarity of temperature, $\mathrm{pH}$, nitrite, phosphate and silicate in surface and

180 bottom water $(\mathrm{P}<0.05)$.

181 Bacterial dominant taxa and alpha-diversity

182 Most of clean sequences (98\%) were assigned into bacterial taxa. We focused our analyses on

183 fourteen taxonomic groups that are greater than $1 \%$ relative abundance in at least one sample.

184 These groups included Alpha-proteobacteria (30.5\%), Gamma-proteobacteria (19.2\%),

185 Bacteroidetes (16.1\%), Cyanobacteria (10.5\%), Actinobacteria (5.8\%), Planctomycetes (3\%),

186 Firmicutes (2.6\%), Rhodothermaeota (2.4\%), Delta-proteobacteria (2.4\%), Beta-proteobacteria

187 (1.8\%), Verrucomicrobia (1.4\%), SAR406 (1\%), Tenericutes (0.4\%), Chloroflexi (0.4\%).

188 Hierarchical clustering analysis (HCA) showed that these abundant phyla/classes can roughly

189 classify samples into surface and bottom clusters, except for P1s and B8s (Fig. 2). Figure 2

190 showed that P1s and B8s were clustered with P1b and B8b, indicating great similarity in these

191 samples. Given that location of P1 and B8 was near the Yellow River estuary (Fig. 1), we

192 consider bacteria in surface and bottom water might be mixing because of the terrestrial runoff.

193 Therefore, it would be better to classify samples from P1 and B8 as a separate cluster (Cluster II).

194 To determine the significant taxa in three groups, a total of 118 bacterial taxa were processed by

195 LEfSe, and 68 taxa were significantly discriminant clusters (Dataset S2). Our results showed (see

196 Fig. S2 and Dataset S2) that the most significant taxa in P1 and B8 (Cluster II) were associated

197 with Bacteroidetes (including class Flavobacteriia, order Flavobacteriales, family

198 Cryomorphaceae and genus Fluviicola), Beta-proteobacteria, Tenericutes (including family 
199 Acholeplasmataceae), and order Oceanospirillales (including family Halomonadaceae and genus 200 Candidatus Portiera). Abundant bacteria in the other surface samples (Cluster I) were related 201 with Cyanobacteria (including genus Synechococcus), Rhodothermaeota (including genus 202 Balneola), and order Rickettsiales. The other bottom samples (Cluster III) were dominated by 203 Planctomycetes (including class Planctomycetia, order Planctomycetales, family 204 Planctomycetaceae and genus Planctomyce), Delta-proteobacteria (including order 205 Desulfobacterales, family Nitrospinaceae, genus Nitrospina and SAR324), SAR406, and order 206 Bacillales (including family Bacillaceae). These discriminant taxa from three groups can be well 207 clustered by their correlation coefficients with spatial factors and environmental parameters (Fig. 208 3). Generally, most of the discriminant taxa from Cluster II exhibited correlations with longitude, 209 water depth, salinity and turbidity $(|\rho|>0.5, \mathrm{P} \leq 0.05$; Dataset S3). Abundant taxa of Cluster I 210 were linked to $\mathrm{pH}$ and turbidity $(|\rho|>0.5, \mathrm{P} \leq 0.05)$, while the dominant bacteria of Cluster III 211 were not only related to these factors, but also strongly affected by collection depth, temperature, 212 dissolved oxygen, nitrate, phosphate and silicate $(|\rho|>0.5, \mathrm{P} \leq 0.05$; Dataset $\mathrm{S} 3)$. Besides, 50 213 abundant taxa accounting for over $50 \%$ of total OTU abundance were evenly dispersed among 214 three clusters. Samples from Cluster I-III shared $70 \%$ of all detected OTUs, and more than $89 \%$ 215 of all OTUs were shared between surface and bottom water samples (Fig. 4). This implied that samples collected in this study shared a large portion of the local bacteria species pool, although some abundant bacteria could be particularly dominant or exclusive in each cluster.

218 Alpha-diversity indexes including observed OTUs, phylogenetic diversity, Chaol and Shannon- 
221 correlations with the physicochemical factors temperature, $\mathrm{pH}$, turbidity, dissolved oxygen, nitrite

222 and silicate $(|\rho|>0.5, \mathrm{P}<0.05$; Table 1). One-way ANOVA indicated Chao1 and Shannon-

223 Wiener indexes of bottom communities were obviously higher than those in surface water $(\mathrm{P}<$

224 0.05; Fig. S3 and Table S1), which implied bacterial communities in bottom water were more

225 diverse than bacterioplankton in surface water. This was consistent with previous studies (Liu et

226 al. 2015; Zinger et al. 2011). The environmental parameter phosphate was linked to the Shannon-

227 Wiener indexes of bottom bacterial communities $(|\rho|>0.5, \mathrm{P}<0.05$; Table 1$)$. As for bacteria in

228 surface water, a general decreasing richness and diversity occurred from P1 to K5, along with

229 decreasing turbidity (Fig. S3, Dataset S1c and Table 1); the parameters longitude, water depth

230 and Chl $a$ were also closely related with alpha-diversity $(|\rho|>0.5, \mathrm{P}<0.05$; Table 1$)$.

231 Microbial distribution in surface and bottom water

232 Using weighted UniFrac distance, we conducted PCoA for all samples. The PCoA plots

233 suggested separation of bacterial communities from surface and bottom water (Fig. 5). The first

234 three principal coordinates PC1, PC2 and PC3 explained $80.89 \%$ of the total variation; PC1 and

235 PC2 (Fig. 5A), together accounting for $69.27 \%$ of the total variation, demonstrated a similar

236 clustering of samples as that in Fig. 2.

237 Mantel and partial Mantel tests were performed to further explore the effects of geographic

238 distance, spatial factors (water depth and collection depth) and physicochemical variables

239 (environment) (Table 2 and Table S2). Our results showed that the beta-diversity of all 14

240 bacterial communities was correlated with collection depth and environment $(\mathrm{P}<0.01$; Table 2$)$,

241 while the distance effect was relatively weak $(\rho=0.18, P>0.05)$. For the beta-diversity of

242 bacterial communities in bottom water, strong effects were mainly derived from geographic 
243 distance, water depth and collection depth $(\rho>0.5, \mathrm{P}<0.05$; Mantel tests $)$, but the correlations

244 between beta-diversity and geographic distance became insignificant when water depth or

245 collection depth was controlled ( $>0.05$; partial Mantel tests). Since collection depth of bottom

246 water was determined as 2-3 $\mathrm{m}$ above seafloor, water depth could be regarded as a factor

247 tantamount to collection depth in shaping community dissimilarity. Hence, water depth was the

248 most important factor generating beta-diversity of bottom bacterial communities. Mantel tests

249 indicated that the beta-diversity of bacterioplankton in surface water was correlated with

250 geographic distance $(\rho<0.5, \mathrm{P}<0.05)$ and environment $(\rho>0.5, \mathrm{P}>0.05)$, while partial Mantel

251 tests suggested their correlations were likely mutually dependent $(|\rho|<0.5, \mathrm{P}>0.05)$.

252 Effect evaluation of each physicochemical parameter revealed that temperature, $\mathrm{pH}$, turbidity,

253 dissolved oxygen, nitrite and silicate was the most important environmental factors influencing

254 the beta-diversity of entire 14 bacterial communities $(\mathrm{P}<0.05$; Mantel tests in Table S2), further

255 confirming the dissimilarity between surface bacterioplankton and bottom bacterial communities.

256 Correlations between temperature, salinity, phosphate and beta-diversity of bottom bacteria $(|\rho|>$

257 0.5, $\mathrm{P}<0.05$; Mantel tests) were observed to be dependent on water depth and collection depth (P

$258>0.05$; partial Mantel tests). As to the bacterioplankton in surface water, community dissimilarity

259 was related to turbidity and nitrite $(|\rho|>0.5, \mathrm{P}<0.05$; Mantel tests in Table S2) without any

260 noticeable dependence (partial Mantel tests).

261 Numbers of pairwise shared OTUs decreased with increasing geographic distance of every two

262 sites in both surface and bottom water, as well as pairwise dissimilarity (Euclidean distance) of

263 water depth, longitude, salinity and phosphate (Fig. S5, Fig. 6 and Table 3), which clearly 
264 suggested the dispersal of bacteria in connective waters were affected and limited by these 265 factors.

266 Discussion

267 Composition of the microbial communities distributed along spatial and environmental 268 gradients

269 We observed horizontal salinity and turbidity gradients ranging from the Bohai Sea to northern 270 Yellow Sea (Fig. S1), while gradients in temperature, salinity, pH, nitrate, phosphate and silicate 271 were reflected in sample collection depths (Dataset S1). Under such condition, the dominant taxa 272 of bacterial communities classified samples into three distinct clusters (Fig. 2 and Fig. 3), 273 demonstrating patterns of bacterioplankton distribution among sampling sites.

274 For instance, genera Synechococcus and Balneola characterized Cluster I, predominated in 275 surface bacterioplankton, and decreased with increasing collection depth (Fig. 3 and Dataset S3). 276 Species in genus Synechococcus require sun light for primary production (Paerl et al. 2012); 277 genus Balneola includes aerobic microbes or facultative anaerobes (Munoz et al. 2016), such as 278 Balneola vulgaris, which has been isolated from surface waters in coastal sea (Urios et al. 2006).

279 Bacterial taxa discriminating Cluster I were likely adapted to surface water conditions as exemplified by the differential abundance of cyanobacterial OTUs and known aerobic heterotroph OTUs in surface samples. Cluster II was dominated by Bacteroidetes (including class Flavobacteriia and genus Fluviicola), Beta-proteobacteria, Tenericutes (including family Acholeplasmataceae), and order

284 Oceanospirillales (including genus Candidatus Portiera) (Fig. 3). Most of these bacterial taxa 
285 showed significantly negative correlationships with longitude (Dataset S3), suggesting the 286 outspread effects from estuarial waters (Campbell \& Kirchman 2013; Liu et al. 2015). Among all 287 these taxa, Flavobacteriia-lineage is related to algal organic matter degradation in the coastal 288 water, which may facilitate the growth of Oceanospirillales (Williams et al. 2013); genus 289 Fluviicola is usually retrieved from river water and riverbeds (Bowman 2014). Our results 290 showed family Acholeplasmataceae was mainly associated with genus Acholeplasma (Dataset 291 S2), species of which are the most common mollicutes in vertebrate animals (Martini et al. 2014). 292 Genus Candidatus Portiera is recognized as the primary endosymbiont of whiteflies (Thao \& 293 Baumann 2004). In our study, these land-source bacteria were dominant and confined in sites P1 294 and B8, consistent with their close proximity to mouth of the Yellow River (van der Gast 2015). Discriminant taxa in Cluster III were Planctomycetes (including genus Planctomyce), Deltaproteobacteria (including genus Nitrospina and SAR324), SAR406, and order Bacillales

297 (including family Bacillaceae) (Fig. 3 and Fig. S2). These taxa were closely related to nutrients carbon, nitrogen, phosphorus and sulfur. For instance, phylotypes of genus Nitrospina are known as nitrite oxidizers, and have been detected in sediments of South Pacific (Durbin \& Teske 2011). SAR324 and SAR406 are typically deep-sea group, and found in aphotic ocean with low-oxygen conditions (Alonso-Saez et al. 2015; Salazar et al. 2016); some of their species are involved in carbon cycling and sulfur oxidation (Sheik et al. 2014). Members of family Bacillaceae perform fundamental roles in degradation of organic matter, nitrification, denitrification, nitrogen fixation and phosphorus solubilization (Mandic-Mulec et al. 2015). As our results indicated, Bacillaceae- 
306 lineage was closely related to $\mathrm{pH}$, dissolved oxygen, nitrite, nitrate, phosphate and silicate (Fig. 3

307 and Dataset S3).

308 In summary, the dominant microbial taxa of each province were greatly affected by spatial

control and environmental conditions, which was consistent with their physiological

requirements, dispersal limitation and ecological functions. Meanwhile a considerable number of

abundant taxa dispersed ubiquitously between surface and bottom layers, and among entire

sampling sites (Dataset S2 and Fig. 4). As Stocker (2012) has reviewed, some microbes actively

exploit environmental heterogeneity, whereas some others just adapt the dynamic

physicochemical gradients. Moreover, each single site has been suggested having a persistent

microbial "seed bank", which can go through ever-shifting environmental conditions over time

(Caporaso et al. 2012). Therefore in our study sea area, strong vertical mixing in winter could

enhance passive dispersal of bacterioplankton through water column, as well as enlarge the

opportunity for microbial seed banks to exchange their species. Those bacteria with broad niche

breadths can widely spread and sustain in the sea water with the help of water movements. When

it comes to summer, stratified seawater shaped distinct environmental conditions in this area,

leading to thriving populations of some bacteria and distinct clustering of microbial communities,

while the samples in surface and bottom (as well as Cluster I-III) still shared a large amount of

OTUs (Fig. 4). We considered this could be an optimal strategy for bacterioplankton to maintain

biodiversity and functions through annual cycles of mixing and stratification in sea water.

\section{Different factors shaped the diversity of bacterial communities in surface and bottom water}

Our results demonstrated geographic distance, spatial factors and physicochemical variables 
328 varied in the surface and bottom communities. For bacterioplankton in surface water, both alpha-

329 and beta-diversity had significant correlations with turbidity (Table 1 and Table S2). Distribution

330 of turbidity was spatially autocorrelated (Dataset S1c); the higher turbidity occurred at P1

331 (Dataset S1b), partly because the Yellow River discharges mixed the surface and bottom water

332 with large amount of sediments along with particles, nutrients and organic matters. High turbidity

333 can cause decrease in light penetration, which explained the significant abundance of

334 photosynthetic bacteria (Cyanobacteria) in surface water of B6-K5 rather than at the estuarial

335 sites P1 and B8 (Fig. 2). In our study, Synechococcus-lineage was the dominant bacteria in

336 surface water, and the most abundant Synechococcus OTU, denovo 4779, was $100 \%$ identical to

337 Synechococcus sp. strain WH 8103 according to BLAST results. Relative abundance of

338 Synechococcus-lineage negatively correlated with ammonium, nitrate and nitrite concentrations,

339 reflecting these microbes preferring lower nutrients in the northern Yellow Sea (Dataset S3).

340 Nitrogen availability (nitrate and nitrite) has been suggested as one factor determining the relative

341 abundances of Synechococcus-lineage in temperate seawater (Choi et al. 2013), whereas our

342 results showed that nitrite $(\rho=-0.56)$ had a strongereffect than nitrate $(\rho=-0.49$, Dataset S3).

343 Additionally, nitrite was another environmental factor greatly affecting beta-diversity of

344 bacterioplankton (Table S2). According to the previous study (Bird \& Wyman 2003), assimilation

345 of nitrate and nitrite in Synechococcus strains can be repressed by high concentrations of

346 ammonium. For instance, ammonium at concentrations above $1 \mu \mathrm{mol} \mathrm{L}{ }^{-1}$ can represses the

347 expression of genes involved in nitrite/nitrate assimilation and inhibits the uptake of nitrate

348 (Lindell et al. 1999; Lindell \& Post 2001). However, Synechococcus sp. strain WH 8103 is

349 capable of assimilating nitrite with the presence of high ammonium concentrations (Wyman \& 
350 Bird 2007). Here we measured the concentrations of ammonium ranged from $0.45 \mu \mathrm{mol} \mathrm{L}^{-1}$ to

$3511.61 \mu \mathrm{mol} \mathrm{L}^{-1}$ in the study area (Dataset S1a). This means nitrate uptake might be inhibited in

352 some sites with ammonium concentrations $>1 \mu \mathrm{mol} \mathrm{L}{ }^{-1}$, while in all surface sites, nitrite

353 assimilation was subject to less inhibition because of the abundant Synechococcus sp. strain WH

354 8103. This could explain the detected result that nitrite was more relative with the bacteria

355 diversity than nitrate.

356 For bacteria in bottom water, community similarity was mainly determined by water depth and

357 collection depth, presenting a depth-decay pattern (Table 2 and Fig. S4). Similar pattern has been

358 observed in coastal and deep-sea sediments, where composition and structure of benthic bacterial

359 community differed significantly with increasing water depth (Jacob et al. 2013; Zinger et al.

360 2011). In our study, water depth varies along the spatial gradient from the shallow coast to

361 northern Yellow Sea. The shallower water depth was near the estuary with mixing of

362 communities from bottom to surface (for example, P1 and B8). With increasing water depth,

363 bacteria in bottom layer were farther from the effects of mixing in estuary. In vertical dimension,

364 we consider collection depth works as a comprehensive proxy for many physicochemical factors

365 (including temperature, salinity and phosphate, Dataset S1d) as reported previously (Fortunato et

al. 2012; Gong et al. 2015; Li et al. 2014).

367 Sea water in the study area is subject to annual circles of winter mixing and summer

368 stratification, and also affected three important water bodies, the Yellow River, Bohai Sea and

369 Yellow Sea, which might lead to the large core bacterial species bank as well as the community

370 distinctions existing in surface and bottom samples (Fig. 4 and Fig. 5). Our results showed both

371 the surface and bottom bacteria were constrained and affected by geographic distance, longitude, 
372 depth, salinity and phosphate (Fig. 6 and Table 3). Phosphate did not exhibit dependence on the 373 spatial factor longitude (Dataset S1); meanwhile the IN/IP ratios in most of samples were much 374 higher than the Redfield's ratio 16 (Redfield 1958), suggesting a phosphate insufficiency for 375 biological communities relative to inorganic nitrogen. Thus we consider that phosphate affecting 376 bacterial dispersal might be unrelated to the spatial reason, and the most possible explanation 377 should be the high ratios of IN to IP.

\section{Conclusion}

379 In this study, we observed significant differences of environmental conditions and a distinct separation of bacterial communities in surface and bottom waters. Vertical separation of bacterial communities has been reported in ocean by numerous studies (Fortunato et al. 2012; Qian et al. 2011; Treusch 2009). This could be explained by stratification of water column as well as vertial distribution of multiple physicochemical factors, such as temperature, salinity, light and nutrients

384 (Fortunato et al. 2012).

385

Now we may answer the questions driving this study: (i) Do the abundant bacteria vary along spatial and physicochemical gradients? Yes. In our study, those discriminant taxa exhibited limited distributions in the study area, while the others presented a ubiquitous distribution. Bacterial distribution along the gradient from the shallow coast to relative open sea shows remarkable horizontal and vertical patterns in bacterial communities. (ii) Do geographic distance and depth contribute more than environmental factors do to spatial variation of microbial communities? Situation differs in bacterial communities form surface and bottom waters. Environmental factors significantly affected the composition and biodiversity of bacterial 
393 communities in surface water. In coastal sea, water depth plays a noticeable role in biogeography

394 of bacteria in bottom water, and leads to a depth-decay pattern of community similarity.

395 Geographic distance enhanced community dissimilarity as previously reported (Martiny et al.

396 2011), and was one of the most important factors driving the spatial variation of microbial

397 communities. However, the only summer samples are not sufficient to uncover the specific

398 mechanisms of their ecological function. To unveil more details, further study is still necessary to

399 integrate time-series data and more complete environmental profile.

400 Acknowledgements

401 The authors acknowledge the editing of English by Prof. Kevin McCartney (University of Maine 402 at Presque Isle) and Prof. Andrzej Witkowski (University of Szczecin). 
403

404

405

406

407

408

409

410

411

412

413

414

415

\section{Reference}

Alonso-Saez L, Diaz-Perez L, and Moran XA. 2015. The hidden seasonality of the rare biosphere in coastal marine bacterioplankton. Environ Microbiol 17:3766-3780.

Bird C, and Wyman M. 2003. Nitrate/Nitrite Assimilation System of the Marine Picoplanktonic Cyanobacterium Synechococcus sp. Strain WH 8103: Effect of Nitrogen Source and Availability on Gene Expression. Appl Environ Microbiol 69:7009-7018.

Bokulich NA, Subramanian S, Faith JJ, Gevers D, Gordon JI, Knight R, Mills DA, and Caporaso JG. 2013. Quality-filtering vastly improves diversity estimates from Illumina amplicon sequencing. Nat Methods 10:57-59.

Bowman JP. 2014. The Family Cryomorphaceae. In: Rosenberg E, DeLong EF, Lory S, Stackebrandt E, and Thompson F, eds. The Prokaryotes: Other Major Lineages of Bacteria and The Archaea. Berlin, Heidelberg: Springer Berlin Heidelberg, 539-550.

Campbell BJ, and Kirchman DL. 2013. Bacterial diversity, community structure and potential growth rates along an estuarine salinity gradient. ISME J 7:210-220.

Caporaso JG, Bittinger K, Bushman FD, Desantis TZ, Andersen GL, and Knight R. 2010a. PyNAST: a flexible tool for aligning sequences to a template alignment. Bioinformatics 26:266-267.

Caporaso JG, Kuczynski J, Stombaugh J, Bittinger K, Bushman FD, Costello EK, Fierer N, Pena AG, Goodrich JK, Gordon JI, Huttley GA, Kelley ST, Knights D, Koenig JE, Ley RE, Lozupone CA, McDonald D, Muegge BD, Pirrung M, Reeder J, Sevinsky JR, Turnbaugh PJ, Walters WA, Widmann J, Yatsunenko T, Zaneveld J, and Knight R. 2010b. QIIME 
Caporaso JG, Paszkiewicz K, Field D, Knight R, and Gilbert JA. 2012. The Western English

Chamberlain SD, Kaplan KA, Modanu M, Sirianni KM, Annandale S, and Hewson I. 2014. Island, Hawai'i. FEMS Microbiol Ecol 89:80-88.

Chen C-TA. 2009. Chemical and physical fronts in the Bohai, Yellow and East China seas.

Durbin AM, and Teske A. 2011. Microbial diversity and stratification of South Pacific abyssal marine sediments. Environ Microbiol 13:3219-3234.

Dang H, Zhou H, Zhang Z, Yu Z, Hua E, Liu X, and Jiao N. 2013. Molecular detection of Candidatus Scalindua pacifica and environmental responses of sediment anammox bacterial community in the Bohai Sea, China. PLoS One 8:e61330.

DeSantis TZ, Hugenholtz P, Larsen N, Rojas M, Brodie EL, Keller K, Huber T, Dalevi D, Hu P, and Andersen GL. 2006. Greengenes, a chimera-checked 16S rRNA gene database and workbench compatible with ARB. Appl Environ Microbiol 72:5069-5072.

Edgar RC, Haas BJ, Clemente JC, Quince C, and Knight R. 2011. UCHIME improves sensitivity and speed of chimera detection. Bioinformatics 27:2194-2200.

Falkowski PG, Fenchel T, and Delong EF. 2008. The microbial engines that drive earth's 
447 Fortunato CS, Herfort L, Zuber P, Baptista AM, and Crump BC. 2012. Spatial variability gradient. ISME J 6:554-563.

Gong J, Shi F, Ma B, Dong J, Pachiadaki M, Zhang X, and Edgcomb VP. 2015. Depth shapes alpha- and beta-diversities of microbial eukaryotes in surficial sediments of coastal ecosystems. Environ Microbiol 17:3722-3737.

Jacob M, Soltwedel T, Boetius A, and Ramette A. 2013. Biogeography of Deep-sea benthic bacteria at regional scale (LTER HAUSGARTEN, Fram Strait, Arctic). PLoS One 8:e72779.

Langenheder S, and Ragnarsson H. 2007. The role of environmental and spatial factors for the composition of aquatic bacterial communities. Ecology 88:2154-2161.

Lee JH, An BW, Bang IW, and Hong GH. 2002. Water and salt budgets for the Yellow Sea. Journal of the Korean Society of Oceanography 37:125-133.

Legendre P, and Legendre LF. 2012. Numerical ecology: Elsevier.

Li J, Li N, Li F, Zou T, Yu S, Wang Y, Qin S, and Wang G. 2014. Spatial diversity of bacterioplankton communities in surface water of northern South China Sea. PLoS One 9:e113014.

Lin X, Xie S-P, Chen X, and Xu L. 2006. A well-mixed warm water column in the central Bohai Sea in summer: Effects of tidal and surface wave mixing. Journal of Geophysical Research 111.

Lindell D, Padan E, and Post A. 1999. Effect of ammonium on nitrate/nitrite uptake and ntcA 
expression in Synechococcus sp. strain WH 7803. Bulletin de l'Institut océanographique:273-278.

Lindell D, and Post AF. 2001. Ecological aspects of ntcA gene expression and its use as an indicator of the nitrogen status of marine Synechococcus spp. Appl Environ Microbiol 67:3340-3349.

Lindstrom ES, and Langenheder S. 2012. Local and regional factors influencing bacterial community assembly. Environmental Microbiology Reports 4:1-9.

Liu J, Fu B, Yang H, Zhao M, He B, and Zhang XH. 2015. Phylogenetic shifts of bacterioplankton community composition along the Pearl Estuary: the potential impact of hypoxia and nutrients. Front Microbiol 6:64.

Mandic-Mulec I, Stefanic P, and van Elsas JD. 2015. Ecology of Bacillaceae. Microbiol Spectrum 3:TBS-0017-2013.

Martini M, Marcone C, Lee I-M, and Firrao G. 2014. The Family Acholeplasmataceae (Including Phytoplasmas). In: Rosenberg E, DeLong EF, Lory S, Stackebrandt E, and Thompson F, eds. The Prokaryotes: Firmicutes and Tenericutes. Berlin, Heidelberg: Springer Berlin Heidelberg, 469-504.

Martiny JB, Bohannan BJ, Brown JH, Colwell RK, Fuhrman JA, Green JL, Horner-Devine MC, Kane M, Krumins JA, Kuske CR, Morin PJ, Naeem S, Ovreas L, Reysenbach AL, Smith VH, and Staley JT. 2006. Microbial biogeography: putting microorganisms on the map. Nat Rev Microbiol 4:102-112.

Martiny JB, Eisen JA, Penn K, Allison SD, and Horner-Devine MC. 2011. Drivers of bacterial beta-diversity depend on spatial scale. Proc Natl Acad Sci U S A 108:7850-7854. 
Munoz R, Rossello-Mora R, and Amann R. 2016. Revised phylogeny of Bacteroidetes and proposal of sixteen new taxa and two new combinations including Rhodothermaeota phyl. nov. Syst Appl Microbiol 39:281-296.

Paerl RW, Turk KA, Beinart RA, Chavez FP, and Zehr JP. 2012. Seasonal change in the abundance of Synechococcus and multiple distinct phylotypes in Monterey Bay determined by rbcL and narB quantitative PCR. Environ Microbiol 14:580-593.

Pernetta JC, and Milliman JD. 1995. Land-ocean interactions in the coastal zone: implementation plan. Oceanographic Literature Review 9:801.

Price MN, Dehal PS, and Arkin AP. 2010. FastTree 2 - approximately maximum-likelihood trees for large alignments. PLoS One 5:e9490.

Qian PY, Wang Y, Lee OO, Lau SC, Yang J, Lafi FF, Al-Suwailem A, and Wong TY. 2011. Vertical stratification of microbial communities in the Red Sea revealed by $16 \mathrm{~S}$ rDNA pyrosequencing. ISME $J$ 5:507-518.

R Development Core Team. 2008. R: A language and environment for statistical computing. . $R$ Foundation for Statistical Computing, Vienna, Austria.

Redfield AC. 1958. The biological control of chemical factors in the environment. American scientist 46:230A-221.

Salazar G, Cornejo-Castillo FM, Benitez-Barrios V, Fraile-Nuez E, Alvarez-Salgado XA, Duarte CM, Gasol JM, and Acinas SG. 2016. Global diversity and biogeography of deep-sea pelagic prokaryotes. ISME J 10:596-608.

Segata N, Izard J, Waldron L, Gevers D, Miropolsky L, Garrett WS, and Huttenhower C. 2011. Metagenomic biomarker discovery and explanation. Genome Biol 12:18. 
512 Sheik CS, Jain S, and Dick GJ. 2014. Metabolic flexibility of enigmatic SAR324 revealed through metagenomics and metatranscriptomics. Environ Microbiol 16:304-317.

Shen F, Suhyb Salama MHD, Zhou Y-X, Li J-F, Su Z, and Kuang D-B. 2010. Remote-sensing 31:2639-2654.

Stocker R. 2012. Marine microbes see a sea of gradients. Science 338:628-633.

Su J. 2001. A review of circulation dynamics of the coastal oceans near China. Acta

van der Gast CJ. 2015. Microbial biogeography: the end of the ubiquitous dispersal hypothesis? Environ Microbiol 17:544-546. 
534

535

536

537
Bacterial biogeography in the coastal waters of northern Zhejiang, East China Sea is highly controlled by spatially structured environmental gradients. Environ Microbiol 17:3898-3913.

Wei H, Sun J, Moll A, and Zhao L. 2004. Phytoplankton dynamics in the Bohai Seaobservations and modelling. Journal of Marine Systems 44:233-251.

Williams TJ, Wilkins D, Long E, Evans F, DeMaere MZ, Raftery MJ, and Cavicchioli R. 2013. The role of planktonic Flavobacteria in processing algal organic matter in coastal East Antarctica revealed using metagenomics and metaproteomics. Environ Microbiol $15: 1302-1317$.

Wyman M, and Bird C. 2007. Lack of control of nitrite assimilation by ammonium in an oceanic picocyanobacterium, Synechococcus sp. strain WH 8103. Appl Environ Microbiol 73:3028-3033.

Xiong J, Ye X, Wang K, Chen H, Hu C, Zhu J, and Zhang D. 2014. Biogeography of the Sediment Bacterial Community Responds to a Nitrogen Pollution Gradient in the East China Sea. Appl Environ Microbiol 80:1919-1925.

Zhang M, Dong Q, Cui T, Xue C, and Zhang S. 2014. Suspended sediment monitoring and assessment for Yellow River estuary from Landsat TM and ETM+ imagery. Remote Sensing of Environment 146:136-147.

Zheng Q, Fang G, and Song YT. 2006. Introduction to special section: Dynamics and Circulation of the Yellow, East, and South China Seas. Journal of Geophysical Research 111.

Zinger L, Amaral-Zettler LA, Fuhrman JA, Horner-Devine MC, Huse SM, Welch DB, Martiny JB, Sogin M, Boetius A, and Ramette A. 2011. Global patterns of bacterial beta-diversity 
in seafloor and seawater ecosystems. PLoS One 6:e24570. 
Figure 1

The map of sampling sites in this study.

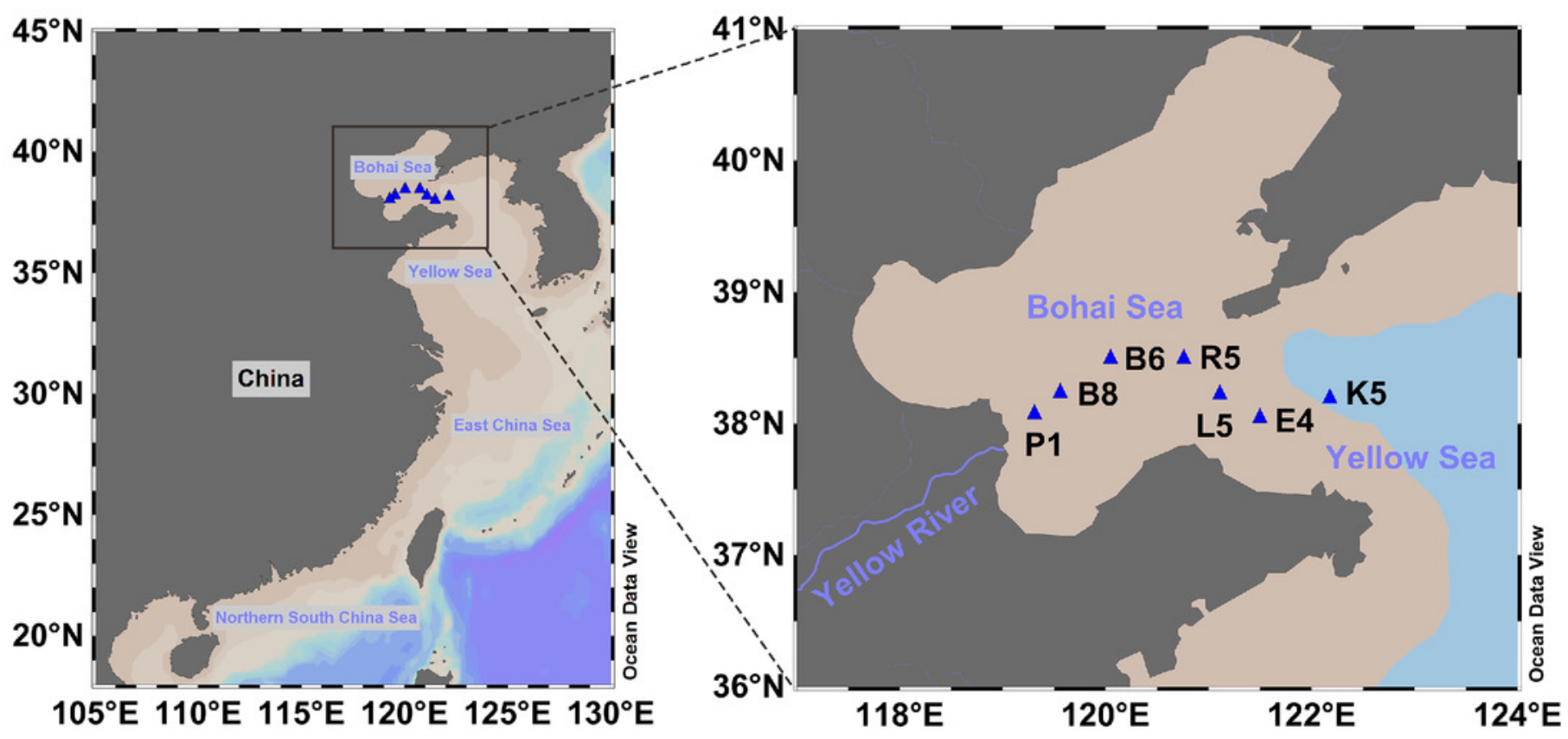


Figure 2

Relative abundance of abundant phyla/classes can classify all samples into distinct clusters.

The abundant taxa are defined with relative abundance $>1 \%$ in at least one sample. The phylum Proteobacteria is shown at class level. Color legend shows the relative abundance of bacterial taxa.

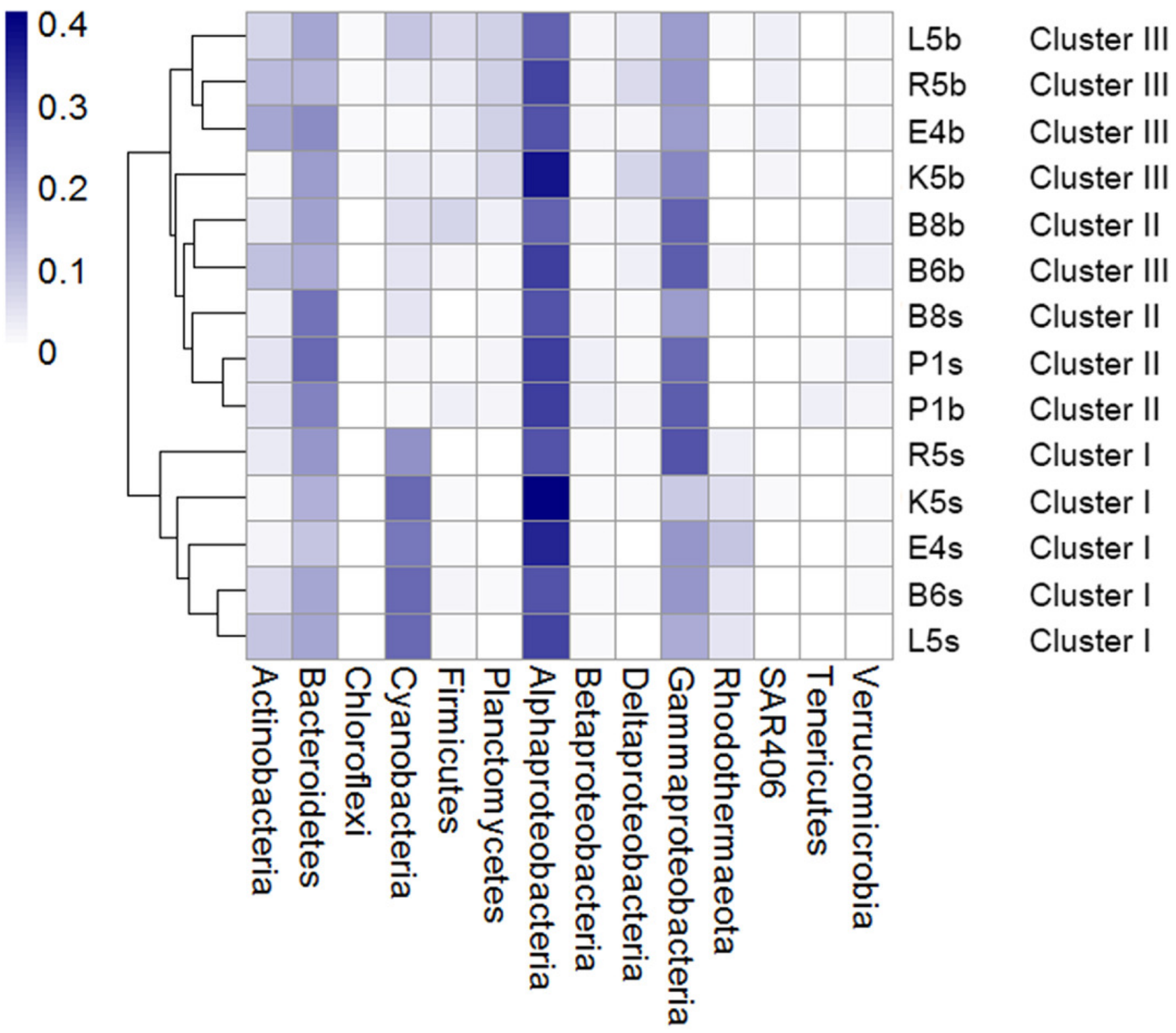


Figure 3

Spearman's correlations between the most significant taxa of each cluster (Fig. 2) and spatial factors, depth, physicochemical variables are shown in this heatmap.

Color legend shows correlation coefficients. More details are shown in Dataset S3. DO: dissolved oxygen; Chl a: chlorophyll a.

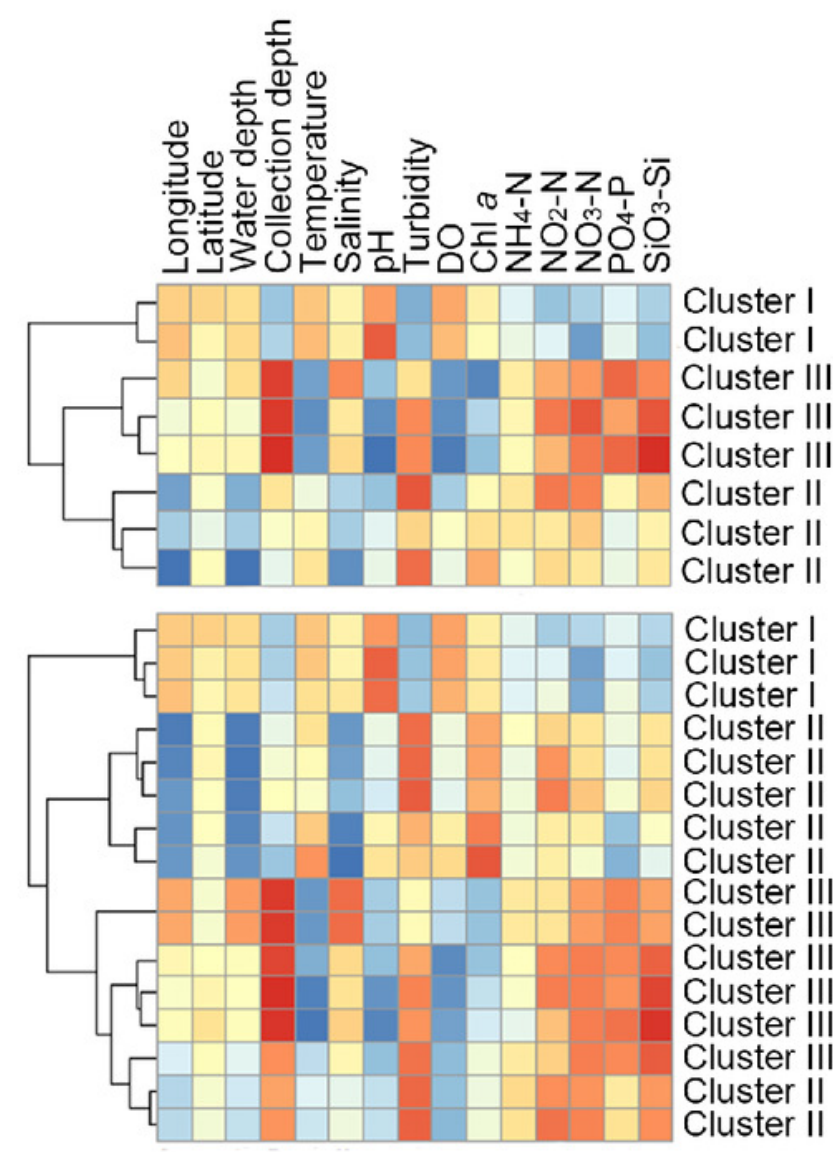

\section{Spearman Correlation Coefficient}

$\begin{array}{lll}-0.5 & 0 & 0.5\end{array}$

Phylum/Class

Family/Genus

Cyanobacteria

Rhodothermaeota

SAR406

Planctomycetes

Deltaproteobacteria

Betaproteobacteria

Bacteroidetes

Tenericutes

Cyanobacteria

Rhodothermaeota

Rhodothermaeota

Tenericutes

Bacteroidetes

Bacteroidetes

Bacteroidetes

Alphaproteobacteria

Deltaproteobacteria

Gammaproteobacteria

Firmicutes

Planctomycetes

Deltaproteobacteria

Gammaproteobacteria

Gammaproteobacteria

Gammaproteobacteria
Synechococcus

Balneolaceae

Balneola

Acholeplasmataceae

Cryomorphaceae

Fluviicola

unknown_Cryomorphaceae

unknown_Kiloniellales

SAR324

unknown_Oceanospirillaceae

Bacillaceāe

Planctomyces

Nitrospina

unknown_Oceanospirillales

Halomonādaceae

Candidatus Portiera 
Figure 4

Venn plots exhibit the OTU numbers shared among Cluster I-III (A), and between surface and bottom communities (B).

A

B

Cluster II

16

6

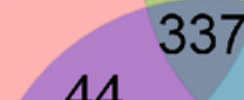

44

28

Cluster III

41
Cluster I

1

Surface

3
Bottom

49 
Figure 5

Spatial variations of bacterial communities are exhibited by principal coordinates analysis (PCoA) with the coordinates PC1 and PC2 (A), and the coordinates PC1 and PC3 (B).

Samples from surface and bottom water are labeled with blue squares and red dots.
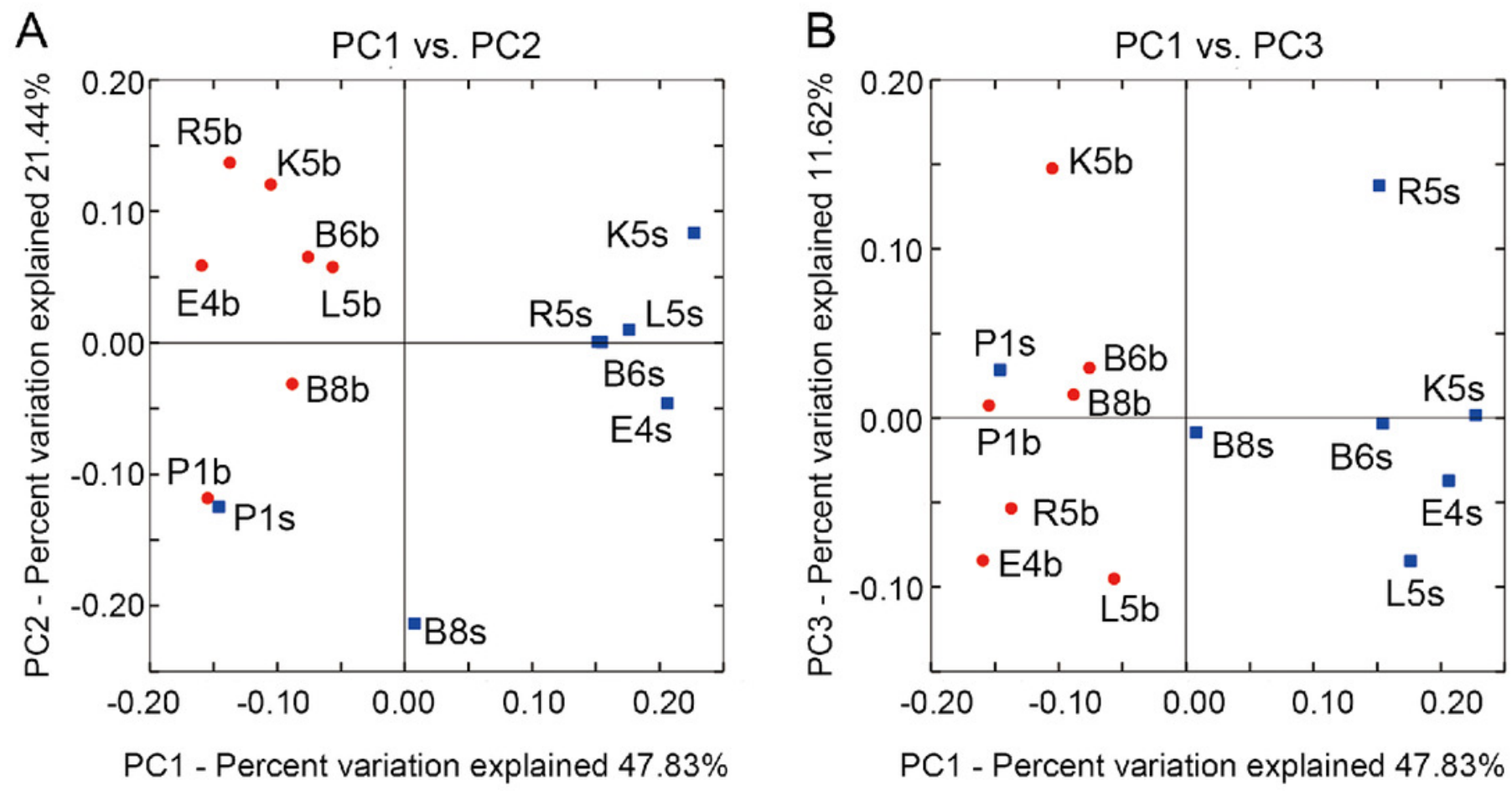


\section{Figure 6}

Relationships between geographic distance, water depth and numbers of pairwise shared OTUs are shown in this figure.

Scatter plots were generated separately for samples from surface (A-B) and bottom water (C-

$D)$. Regression lines, along with regression coefficients $(R)$ and probability $(P)$, were generated using general linear model (GLM).

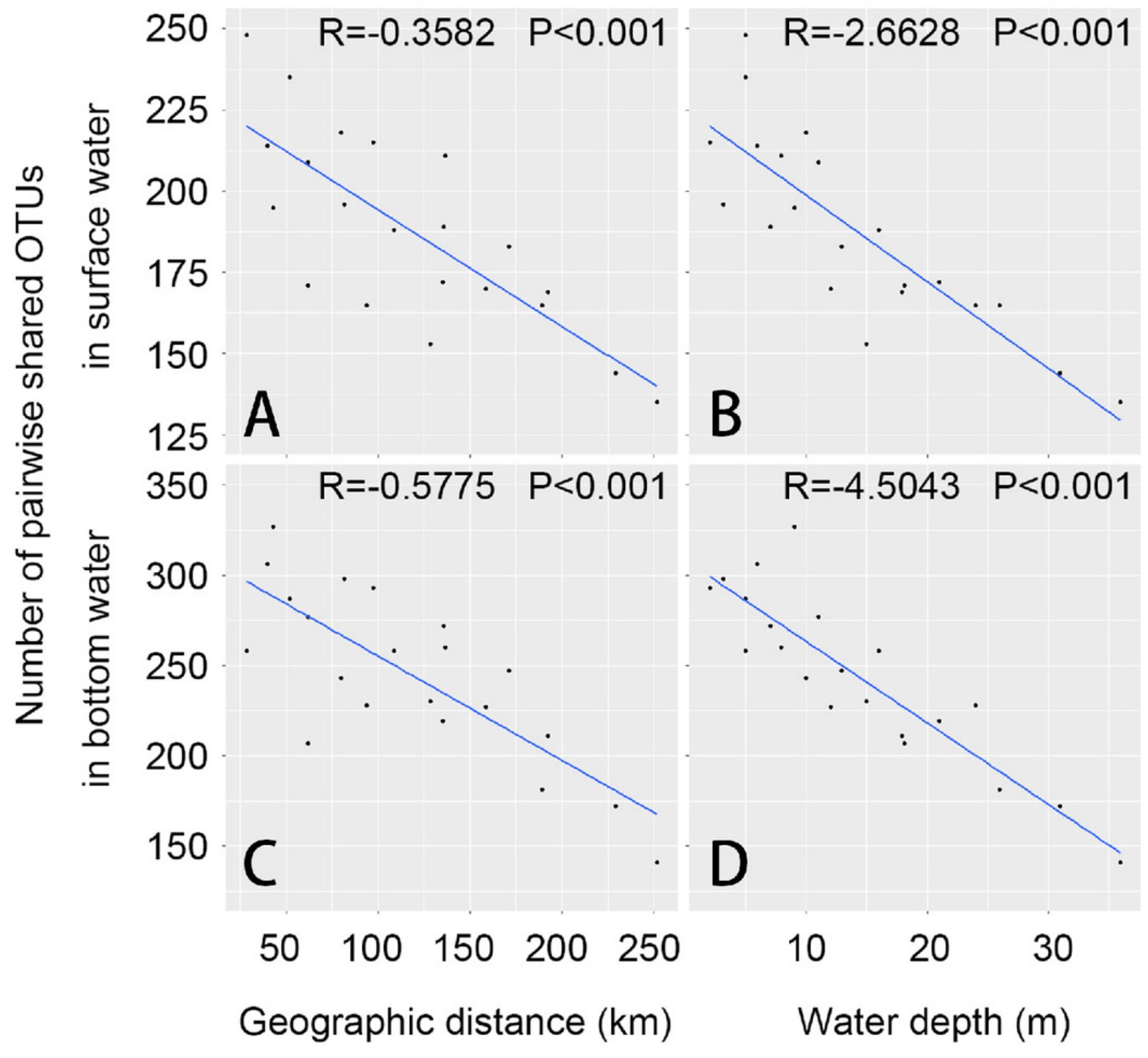




\section{Table $\mathbf{1}$ (on next page)}

Spearman's correlations between spatial factors, physicochemical parameters and alpha-diversity indexes.

Correlation tests were performed separately using all samples, samples from surface water and bottom water. Significant correlations are shown in this table, and highlighted with bold type ( $P \leq 0.05$ after Benjamini-Hochberg multiple test correction). Correlations with $|\rho| \geq 0.5$ are labeled with gray shadow. Chl a: chlorophyll a. 
Table 1 Spearman's correlations between spatial factors, physicochemical parameters

and alpha-diversity indexes. Correlation tests were performed separately using all samples, samples from surface water and bottom water. Significant correlations are shown in this table, and highlighted with bold type ( $\mathrm{P} \leq 0.05$ after Benjamini-Hochberg multiple test correction). Correlations with $|\rho| \geq 0.5$ are labeled with gray shadow. Chl $a$ : chlorophyll a.

\begin{tabular}{llrrrr}
\hline Samples & Variables & $\begin{array}{c}\text { Observed } \\
\text { OTUs }\end{array}$ & $\begin{array}{r}\text { Phylogenetic } \\
\text { Diversity }\end{array}$ & Chao1 & Shannon-Wiener \\
\hline \multirow{2}{*}{ All samples } & Temperature & $-\mathbf{0 . 6 2}$ & $-\mathbf{0 . 6 1}$ & -0.51 & -0.47 \\
& $\mathrm{pH}$ & $-\mathbf{0 . 6 1}$ & $-\mathbf{0 . 6 5}$ & -0.49 & $-\mathbf{0 . 6 6}$ \\
& Turbidity & $\mathbf{0 . 7 0}$ & $\mathbf{0 . 7 2}$ & $\mathbf{0 . 7 0}$ & $\mathbf{0 . 8 6}$ \\
& Dissolved oxygen & $-\mathbf{0 . 7 1}$ & $-\mathbf{0 . 7 0}$ & $-\mathbf{0 . 6 6}$ & $\mathbf{- 0 . 7 9}$ \\
& $\mathrm{NO}_{2}$-N & $\mathbf{0 . 7 6}$ & $\mathbf{0 . 7 1}$ & $\mathbf{0 . 7 8}$ & $\mathbf{0 . 7 4}$ \\
& $\mathrm{NO}_{3}-\mathrm{N}$ & 0.42 & 0.44 & 0.24 & $\mathbf{0 . 6 7}$ \\
& $\mathrm{SiO}_{3}$-Si & $\mathbf{0 . 6 3}$ & $\mathbf{0 . 6 6}$ & $\mathbf{0 . 5 7}$ & $\mathbf{0 . 7 4}$ \\
\hline \multirow{2}{*}{ Samples from } & Longitude & $-\mathbf{0 . 7 9}$ & $-\mathbf{0 . 7 9}$ & $-\mathbf{0 . 7 5}$ & $\mathbf{- 0 . 9 3}$ \\
surface water & Water Depth & $\mathbf{- 0 . 8 9}$ & $-\mathbf{0 . 8 9}$ & $-\mathbf{0 . 8 6}$ & $\mathbf{- 0 . 8 2}$ \\
& $\mathrm{Salinity}$ & -0.63 & -0.63 & -0.63 & $\mathbf{- 0 . 8 8}$ \\
& $\mathrm{pH}$ & -0.68 & -0.68 & -0.64 & -0.75 \\
& Turbidity & $\mathbf{0 . 7 9}$ & $\mathbf{0 . 7 9}$ & $\mathbf{0 . 8 6}$ & $\mathbf{0 . 9 3}$ \\
& $\mathrm{Chl} a$ & $\mathbf{0 . 7 9}$ & $\mathbf{0 . 7 9}$ & $\mathbf{0 . 8 9}$ & 0.5 \\
\hline Samples from & $\mathrm{PO}_{4}-\mathrm{P}$ & -0.40 & -0.46 & -0.29 & $\mathbf{- 0 . 8 6}$ \\
bottom water & & & & & \\
\hline
\end{tabular}




\section{Table 2 (on next page)}

Mantel and partial Mantel tests for the Spearman's rank correlations between geographic distance, water depth, collection depth, environment and beta-diversity.

Tests were performed separately using all samples, samples from surface water and bottom water. Correlations are shown in this table and highlighted with gray shadow when $|\rho| \geq 0.5$. When $P$ values are less than 0.05 after Benjamini-Hochberg multiple test correction, correlations are shown with bold type. Environment was calculated as Euclidean distances of physicochemical parameters. 
Table 2 Mantel and partial Mantel tests for the Spearman's rank correlations between geographic distance, water depth, collection depth, environment and beta-diversity. Tests were performed separately using all samples, samples from surface water and bottom water. Correlations are shown in this table and highlighted with gray shadow when $|\rho| \geq 0.5$. When $P$ values are less than 0.05 after Benjamini-Hochberg multiple test correction, correlations are shown with bold type. Environment was calculated as Euclidean distances of physicochemical parameters.

\begin{tabular}{|c|c|c|c|c|c|c|}
\hline \multirow[t]{2}{*}{ Samples } & \multirow[t]{2}{*}{ Variables } & \multirow[t]{2}{*}{$\begin{array}{l}\text { Mantel } \\
\text { tests }\end{array}$} & \multicolumn{4}{|c|}{$\begin{array}{c}\text { Partial Mantel tests } \\
\text { control factors }\end{array}$} \\
\hline & & & $\begin{array}{c}\text { Geographi } \\
\text { c } \\
\text { distance } \\
\end{array}$ & $\begin{array}{l}\text { Water } \\
\text { depth }\end{array}$ & $\begin{array}{l}\text { Collection } \\
\text { depth }\end{array}$ & Environment \\
\hline \multirow[t]{4}{*}{ All samples } & $\begin{array}{l}\text { Geographic } \\
\text { distance }\end{array}$ & 0.18 & - & 0.10 & 0.19 & 0.18 \\
\hline & Water depth & 0.16 & 0.04 & - & 0.12 & 0.13 \\
\hline & Collection depth & 0.44 & 0.45 & 0.43 & - & 0.35 \\
\hline & Environment & 0.30 & 0.29 & 0.28 & 0.08 & - \\
\hline \multirow[t]{4}{*}{$\begin{array}{l}\text { Samples from } \\
\text { surface water }\end{array}$} & $\begin{array}{l}\text { Geographic } \\
\text { distance }\end{array}$ & 0.49 & - & 0.47 & - & 0.35 \\
\hline & Water depth & 0.21 & -0.13 & - & - & 0.26 \\
\hline & Collection depth & -0.08 & 0.07 & -0.05 & - & -0.01 \\
\hline & Environment & 0.57 & 0.47 & 0.58 & - & - \\
\hline \multirow[t]{4}{*}{$\begin{array}{l}\text { Samples from } \\
\text { bottom water }\end{array}$} & $\begin{array}{l}\text { Geographic } \\
\text { distance }\end{array}$ & 0.52 & - & 0.09 & 0.08 & 0.53 \\
\hline & Water depth & 0.78 & 0.68 & - & 0.17 & 0.76 \\
\hline & Collection depth & 0.77 & 0.67 & -0.02 & - & 0.76 \\
\hline & Environment & 0.34 & 0.37 & 0.19 & 0.34 & - \\
\hline
\end{tabular}




\section{Table 3 (on next page)}

Spearman's correlations between the numbers of pairwise shared OTUs and pairwise Euclidean distances of multiple variables.

Correlation tests were performed separately using samples from surface water and bottom water. Significant correlations are shown in this table and highlighted with gray shadow when $|\rho| \geq 0.5$. When $P$ values are less than 0.05 after Benjamini-Hochberg multiple test correction, correlations are shown with bold type. 
Table 3 Spearman's correlations between the numbers of pairwise shared OTUs and pairwise Euclidean distances of multiple variables. Correlation tests were performed separately using samples from surface water and bottom water. Significant correlations are shown in this table and highlighted with gray shadow when $|\rho| \geq 0.5$. When $P$ values are less than 0.05 after Benjamini-Hochberg multiple test correction, correlations are shown with bold type.

\begin{tabular}{lcc}
\hline Variables & $\begin{array}{c}\text { Samples from } \\
\text { surface water }\end{array}$ & $\begin{array}{c}\text { Samples from } \\
\text { bottom water }\end{array}$ \\
\hline Geographic distance & $\mathbf{- 0 . 7 2}$ & $\mathbf{- 0 . 6 7}$ \\
Water depth & $-\mathbf{0 . 8 7}$ & $-\mathbf{0 . 8 7}$ \\
Longitude & $-\mathbf{0 . 7 4}$ & $-\mathbf{0 . 6 9}$ \\
Temperature & -0.21 & $\mathbf{- 0 . 8 9}$ \\
Salinity & $-\mathbf{0 . 8 2}$ & $\mathbf{- 0 . 8 1}$ \\
$\mathrm{PO}_{4}-\mathrm{P}$ & $-\mathbf{0 . 5 5}$ & $\mathbf{- 0 . 4 9}$ \\
\hline
\end{tabular}

